\title{
Research on Information Feedback Regulation System of Basketball Training Based on ANT Dynamic Theory
}

\author{
Yun Wang ${ }^{1}$, Guimin Dai ${ }^{2}$ \\ ${ }^{1}$ Faculty of Sports Training, Hebei Institute of Physical Education, Shijiazhuang, China \\ w_angyun@126.com \\ ${ }^{2}$ Faculty of Sports Art, Hebei Institute of Media, Shijiazhuang, China
}

\begin{abstract}
Keywords: information control system; feedback regulation; random variable, generalized linear system; origin drawing
\end{abstract}

\begin{abstract}
The basketball training is a long-term process of repeatedly. If you can master the feedback information the training process in time and make adjustment of the sports and sports exercise intensity, it can greatly improve the basketball training effect and reduce training investment and increase the economic benefit of sports training. This paper establishes ANT dynamic metabolic model according to the body metabolism of ANT theory in the basketball training process and establishes training control information system model according to feedback control principle of the generalized linear system. This paper monitors the ANT dynamic metabolic process of project jogging, touch line, layup and physical confrontation in the process of basketball training. This paper uses professional drawing software-origin to process data chart obtains the parameters optimization of the same sport after information control system regulation which realizes the automation control of information in basketball movement process. Finally, this paper summarizes the drawback of the system which provides the theoretical basis for the development of basketball training.
\end{abstract}

\section{Introduction}

Due to the basketball movement is the movement of heated confrontation, and basketball training process is a complicated and repeatedly process. If the intensity of training exercise and sport is too small, it can not reach the training effect. If the amount of exercise is too big, it can cause the muscle fatigue damage. Choosing a suitable method to determine the basketball training exercise and strength is the expectation of basketball coaches[1]. This paper develops a basketball training information control system which can monitor dynamic metabolic process of biological metabolism ANT of basketball player in the training process real-timely to adjust training programs and training exercise and sport intensity[2,3]. The first part of this paper introduces the dynamic model based on ANT metabolic process and information feedback control system based on the generalized linear system and gives the flow chart. The second part of this paper test the model and give the contrast table of training parameters optimization before and after[4,5]. It summarizes the system deficiency through the data analysis and provides a theoretical reference for the construction of basketball training information control system.

\section{The feedback control system model of basketball training information control based on ANT}

In the basketball training process and with the movement, the ANT metabolic process of internal body is a dynamic balance process[6-8]. It can be used to measure the response of body on the input of motion signal, and the amount of ANT metabolism can measure movement effect and extent of body muscle organs to the movement. This valuable information can provide the reference to coaches after information feedback and adjust basketball training and sports training.

First, assuming that in basketball training process, ANT which participates in the metabolism and did not attends the metabolism is a random variable. Assuming that the mount of ANT which participates in the metabolism in time $\mathrm{x}$ is $\mathrm{T}$, and the actual metabolism is T. The ANT enters into the working state new is $\mathrm{u}$. And assuming that the probability of $\mathrm{T}=\mathrm{t}$ is $\mathrm{R}(\mathrm{x})$, so the number of ANT which new entry and stop working can be represented as[9]:

$$
R_{n}(x)\left(1-u \Delta x-\frac{t}{u} \Delta x\right)
$$


Metabolic number is $\mathrm{t}-1$, and the number which starts to metabolize is:

$$
R_{n-1}(x) u \Delta x
$$

When $\Delta x \rightarrow 0$, the probability of the occurrence of two or more than two metabolic state changes is zero, and we can get difference equation[10]:

$$
R_{n}(x+\Delta x)=R_{n}(x)\left(1-u \Delta x-\frac{t}{u} \Delta x+R_{n-1}(x) u \Delta x\right.
$$

So we can set the partial differential equation as:

$$
\frac{d R_{n}(x)}{d x}=-\left(u+\frac{t}{u}\right) R_{n}(x)+R_{n-1}(x) u
$$

We can define the generalized basketball training information system as follows[11]:

$$
\left\{\begin{array}{c}
E t=D t+F u+Q a \\
A=v a \\
f=B t+P a
\end{array}\right.
$$

$\mathrm{T}$ is time control vector, and $\mathrm{u}$ is input estimation parameter, and $\mathrm{f}$ is error vector value, and $\mathrm{E}$ is result, DQABP is coefficient matrix, and we can get information feedback controller as[12]:

$$
\mathrm{U}=\mathrm{gt}+\mathrm{ma}
$$

We can get the basketball training information control system which is shown in Figure 1.
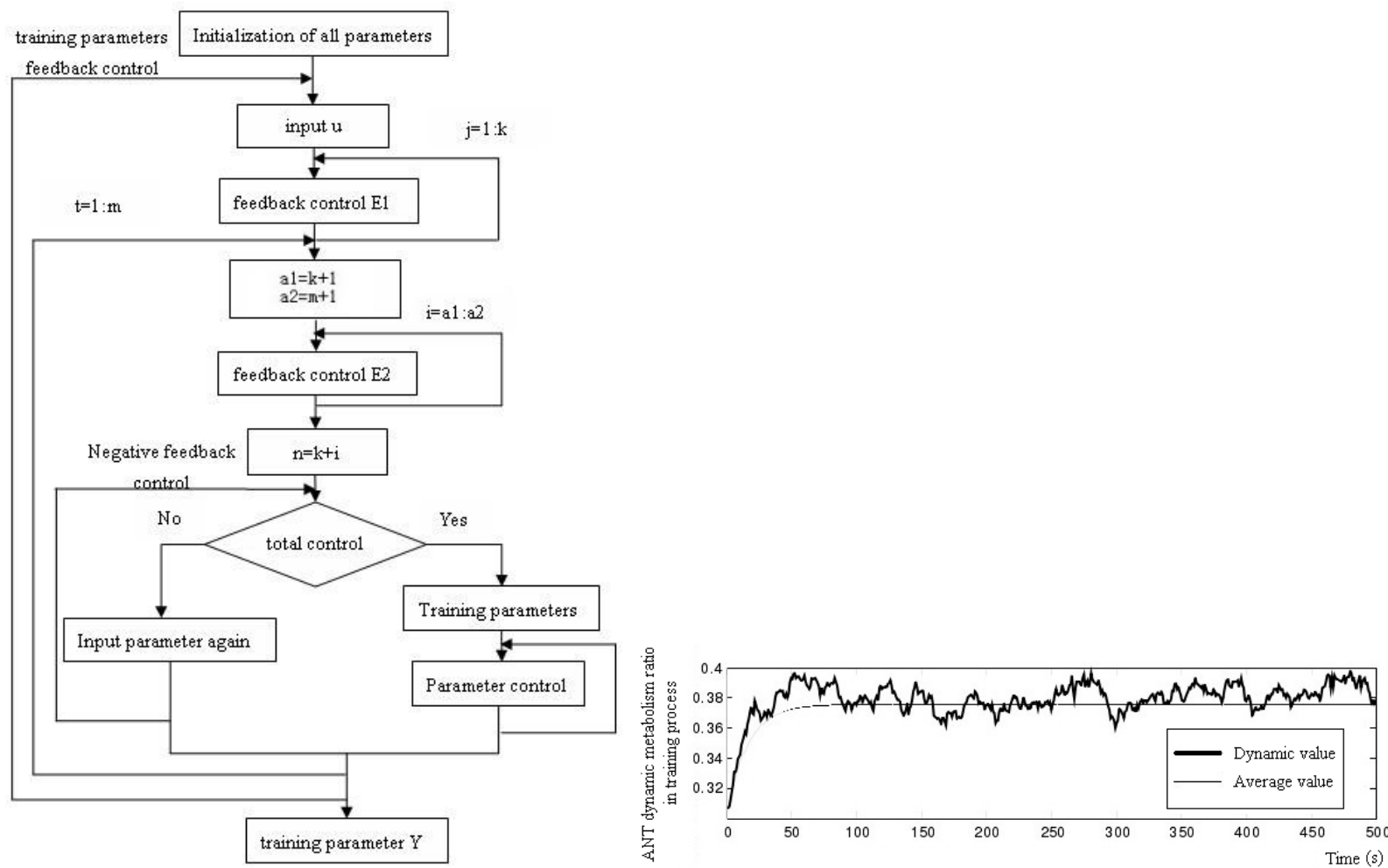

Figure 1. Schematic diagram of basketball training information control system Figure2 ANT dynamic metabolism ratio of jogging training

The parameter $\mathrm{u}$ is the quantity of ANT into the work state of the mitochondria cell modules in the basketball training process and through the electron monitoring and in the unit time. In the process of information feedback we can set $u$ value which is the initial assessment shown in the following Table 1.

According to the setting of standard parameters, we can calculate ANT dynamic metabolism ratio of the basketball training process which can determine the training and sports strength in the training process.

TABLE I.
\begin{tabular}{|l|l|l|l|l|}
\hline \multicolumn{1}{|c|}{$\mathbf{u 1}$} & \multicolumn{1}{c|}{ ESTIMATION OF DYNAMIC SIMULATION U VALUE } \\
\hline $9.02 \times 106$ & $10.13 \times 106$ & $11.25 \times 106$ & $13.08 \times 106$ & $9.75 \times 106$ \\
\hline u6 & u7 & u8 & u 9 & u10 \\
\hline $15.36 \times 106$ & $9.86 \times 106$ & $12.53 \times 106$ & $17.25 \times 106$ & $16.13 \times 106$ \\
\hline
\end{tabular}




\section{Effect analysis on information control system of basketball training}

Through the first part of the basketball control information system model, we can test the ANT metabolic changes in basketball training process. Through the electron microscope scanning, we can monitor the number of ANT which participate into the metabolism and then react it to information control system for the calculation and get the latest training effect parameter after the optimization of the parameters[13]. Origin is powerful professional drawing software. We can use the Origin to drawn the result data of a lot of times for observation.

First of all, we can confirm training programs and physical activities of basketball preliminary which is shown in Table 2.

TABLE II. THE PRELIMINARY SURE TABLE OF BASKETBALL TRAINING SPORTS AND
\begin{tabular}{|l|l|l|}
\hline $\begin{array}{c}\text { training } \\
\text { programs }\end{array}$ & $\begin{array}{c}\text { the number before } \\
\text { fifty seconds }\end{array}$ & $\begin{array}{c}\text { total number of } \\
\mathbf{5 0 0} \text { seconds }\end{array}$ \\
\hline jogging & 5 rings & 40 \\
\hline touch line & 10 & 80 \\
\hline layup & 5 & 45 \\
\hline $\begin{array}{l}\text { Against the } \\
\text { body }\end{array}$ & 8 & 50 \\
\hline
\end{tabular}

Table 2 is basketball project and basketball training exercise parameter according to former basketball training experience. In the training process we can monitor and get jogging ANT metabolic change curve which is shown in Figure 2.

From Figure 2 we can see that the ANT dynamic metabolism ratio of training process increase at the beginning, and become more and more stable after about fifty seconds which is coincide with the ANT theory metabolism data in the movement process[14]. We can draw the ANT dynamic metabolic process of four sports using Origin software which is shown in Figure 3.
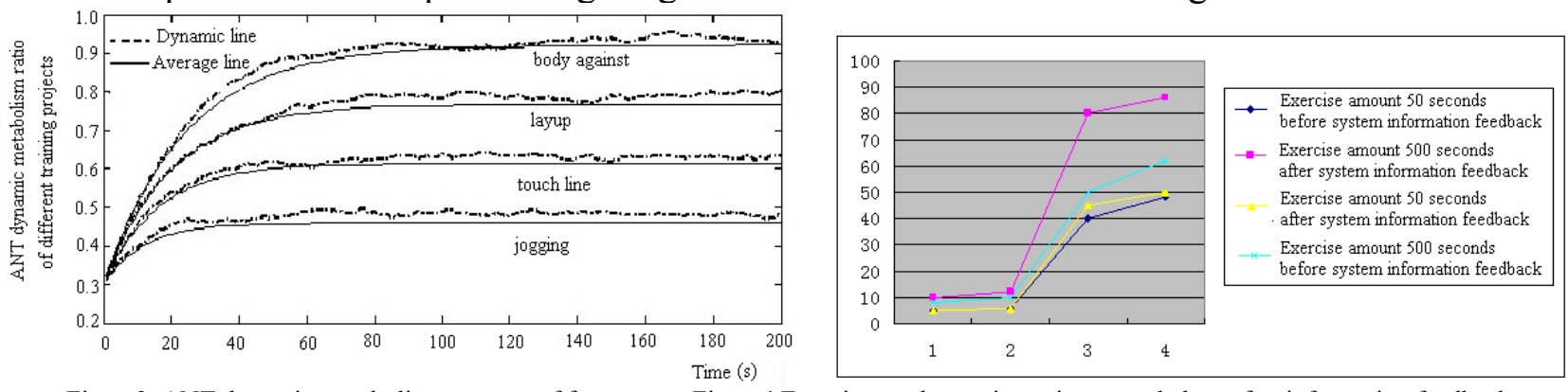

Figure3. ANT dynamic metabolic processes of four sports Figure4.Exercises and sport intensity control chart after information feedbacks

Through the Figure 3, we can observe that at the beginning of four sports the ANT metabolic rate is relatively small, and become more and more stable after metabolism about fifty seconds. The number of ANT jogging dynamic metabolism is the fewest; and the ANT dynamic metabolism in the process of body against is the strongest[15]. The number of ANT metabolism in dynamic metabolic processes is higher than experience number. We can substitute this information into training control system and get optimization data such as Table 3 and Figure 4 through the feedback control:

TABLE III. \begin{tabular}{|l|l|l|}
\hline $\begin{array}{c}\text { THE EXERCISES AND SPORT INTENSITY CONTROL AFTER INFORMATIO } \\
\text { programs }\end{array}$ & $\begin{array}{c}\text { the number before } \\
\text { fifty seconds }\end{array}$ & $\begin{array}{c}\text { total number of 500 } \\
\text { seconds }\end{array}$ \\
\hline jogging & 6 rings & 48 \\
\hline touch line & 12 & 86 \\
\hline layup & 6 & 50 \\
\hline $\begin{array}{l}\text { Against the } \\
\text { body }\end{array}$ & 10 & 62 \\
\hline
\end{tabular}

Through the feedback control of basketball training information control system, we can find that when the basketball sports' condition is constant, physical activity before and after basketball information system control is change. The physical activity is increasing first fifty seconds and the five hundred seconds of information feedback which corresponding to the theory of the number of metabolism ANT time in the graph is higher than the theoretical value which explain reliability and stability of the system.

\section{The deficiency of information control system}

In the case analysis of the second part of the basketball training information control system, we can find the information control system operation results of this paper is consistent with actual conditions which proves the stability and effectiveness of information control system. But there are also many deficiencies in the system.

(1) supervision of feedback regulation 
The information control system feedback control of this paper is only involves four projects which will inevitably cause the adaptability of different project systems. The future research should increase the monitoring strength of feedback control, and monitors more sports training projects and ANT feedbacks of athletes in training process.

(2) Innovation of basketball sports

The information system inspection items of this paper are created based on past experiences which have no innovation on basketball projects and no research on the effect of the system on the basis of the innovation project which has some limitations of new system identification.

(3)The effect evaluation of training information system

Training information system has not yet set up a comprehensive performance evaluation system. So there is no further inspection of the comprehensive stability and effect of system.

\section{Conclusion}

This paper established the basketball training information control system based on the ANT metabolic process of basketball training process and the theory of generalized linear information control system. It given ANT dynamic metabolic mathematical model and generalized linear system matrix model in the first part of this paper and established the basketball training information control system flow chart through the model and been tested in the second part. In the inspection process, it compared the actual training ANT monitoring dynamic metabolic value and experience value and got the final training parameters optimization by substituted the results into information control system. Origin chart analysis can find that the training exercise after optimized was slightly increased than before which is corresponding with the ANT metabolism. Research on the basketball training information control system research related to the quality and effect of basketball training. We should further analyze and research the better, faster and more stable information control algorithm.

\section{References}

[1] Daizhan Cheng, Yali Dong. Output mediation and principle of inner membrane. Journal of automation, 2009,29(02):284-295

[2] Chunhua Yi. Research status and development trend of decision support system . Decisionmaking reference.2012,5(02):23-34.

[3] Huacheng Xue. Management information system.Tsinghua university press,2013(01):264-265.

[4] YunLiang Wang, FengHua Shao, ping li, JiYu Lou. Mitochondrial disease Chinese practical nerve disease magazine, 2009, 10(18):137-140.

[5] Ming Lou, Wei Cui, Yi Zhang, Zhi Liu; The design and implementation of diver sports training management information system.Computer engineering and application,2011(16):23-24.

[6] Qingzhu Sun, Lan Wen, RuCong Huang, Ping Chen, research and development of rowing athletes' ability diagnostic evaluation and control training decision support system. Shandong sports university,2012(02):23-34.

[7] Wei Bian, Li Huang. Design and implementation of professional sports team information management system .journal of sports science,2012 (03):34-40.

[8] Jie Bai. Application situation and prospect of sports information technology .journal of sports culture guide. 2011 (09):45-50.

[9] Zhenxiang Zeng, Jinluan Ren, Min Zhang, Decision support center--- the development trend of DSS . Journal of systems engineering and electronics, 2010 (02):23-34.

[10] Yongquan Zheng. Theory research of basketball training information control system. sports science research,2009(01):34-35.

[11] Caixia Zhang, Jiandong Zhu. Output regulation problem of linear singular system.Chinese control conference proceedings, dalian, Liaoning, 2001(03):31-36.

[12] Hebin Wu.Main factors of sustainable development of Shanxi Province's high school basketball training. Journal of Beijing sports university, 2007 (01):23-25.

[13] Shaohua Wang. Research on the professional quality training of youth basketball athletes. Journal of Nanjing institute of physical education (natural science edition), 2009(01):34-35.

[14] Fanjing Meng. Long-term impact of training way on young male basketball players. Journal of Maoming College, 2009(06):23-25.

[15] Hui Hu. Value of basketball in university students' leisure sports . Journal of Shangrao normal college ,2010(03):34-35. 